Published in final edited form as:

Hum Brain Mapp. 1997 ; 5(4): 238-242. doi:10.1002/(SICI)1097-0193(1997)5:4<238::AID-

HBM6>3.0.CO;2-4.

\title{
Automated Labeling of the Human Brain:
}

\section{A Preliminary Report on the Development and Evaluation of a Forward-Transform Method}

J.L. Lancaster ${ }^{1,{ }^{*}, \text { L.H. Rainey }}{ }^{1}$, J.L. Summerlin ${ }^{1}$, C.S. Freitas ${ }^{1}$, P.T. Fox ${ }^{1}$, A.C. Evans ${ }^{2}$, A.W. Toga ${ }^{3}$, and J.C. Mazziotta ${ }^{3}$

${ }^{1}$ Research Imaging Center, University of Texas Health Science Center at San Antonio, San

Antonio, Texas 78284-6240

${ }^{2}$ Montreal Neurological Unit, Magill University, Montreal, Quebec, Canada H3A 2B4

${ }^{3}$ Laboratory of Neurological Imaging, University of California at Los Angeles, Los Angeles, California 90024-1769

\section{Abstract}

A forward-transform method for retrieving brain labels from the 1988 Talairach Atlas using $x-y-z$ coordinates is presented. A hierarchical volume-occupancy labeling scheme was created to simplify the organization of atlas labels using volume and subvolumetric components.

Segmentation rules were developed to define boundaries that were not given explicitly in the atlas. The labeling scheme and segmentation rules guided the segmentation and labeling of 160 contiguous regions within the atlas. A unique three-dimensional (3-D) database label server called the Talairach Daemon (http://ric.uthscsa.edu/projects) was developed for serving labels keyed to the Talairach coordinate system. Given an x-y-z Talairach coordinate, a corresponding hierarchical listing of labels is returned by the server. The accuracy and precision of the forwardtransform labeling method is now under evaluation.

\section{Keywords}

spatial normalization; segmentation; Talairach Atlas; Talairach Daemon; naming hierarchy; brain labeling

\section{INTRODUCTION}

Standardized three-dimensional (3-D) coordinates based on the Talairach Atlas [Talairach and Tournoux, 1988] are used with increasing frequency for analyzing and reporting human neuroimaging research. Such coordinates provide precise, quantitative descriptions of locations within an atlas enabling direct and consistent access to its anatomical labels [Fox, 1995]. There are two, literally opposite, coordinate-based methods to retrieve brain labels from an atlas for a brain image. Both rely on spatial normalization to transform a brain image volume to conform with features of a brain atlas. In the first, or forward-transform method, brain coordinates are used as indices to locations within a brain atlas to retrieve a corresponding label (coordinatelable mapping). The forward method is common practice when using the 1988 Talairach Atlas, the Brain-Map ${ }^{\mathrm{TM}}$ database [Fox et al., 1994], or other brain atlases [Roland et al., 1994] to get labels. For the second, or inverse-transform method,

(C) 1997 Wiley-Liss. Inc.

* Correspondence to: Jack L. Lancaster, Biomedical Image Analysis Division, Research Imaging Center, University of Texas Health Science Center at San Antonio, 7703 Floyd Curl Drive, San Antonio, TX 78284-6239. 
volumes of interest (VOIs) are transformed from an atlas to conform to a brain image volume (label-coordinate mapping). This method is used by the ANIMAL software developed at Montreal Neurological Institute [Collins et al., 1995] and has been implemented in various formats by others.

Each labeling strategy relies on accurate segmentation of labeled structures in an atlas brain, and both are under development as part of the International Consortium for Brain Mapping (ICBM) project [Mazziotta et al., 1995]. The forward method is based on the 1988 Talairach Atlas where the segmentation task is simplified, since labels already exist. However, boundaries are not well-defined for all labeled structures. The inverse method is based on an individual highresolution MRI brain image volume that was transformed to conform to a standard atlas, and serves as a 3-D surrogate for the original atlas. Segmentation within this atlas volume would be impossible for smaller structures such as Brodmann areas without dependence on other atlases. Neither spatial normalization nor atlas segmentation can be performed with complete accuracy, though spatial normalization is considered to be more correct when using a surrogate atlas with characteristics similar to the subject brain image [Collins et al., 1994; Friston et al., 1995], favoring the inverse method. Conversely, segmentation is more complete using a published atlas, since many more brain regions are available, favoring the forward method. These factors led to the study and development of both methods, a forward method based on the 1988 Talairach Atlas and an inverse method based on the atlas concept of ANIMAL. This preliminary report describes the labeling scheme and segmentation rules for a forward-transform method and its initial implementation for anatomical labeling.

\section{MATERIALS AND METHODS}

\section{Talairach Atlas labeling scheme}

A volume-filling, hierarchical, anatomical-labeling scheme was used wherein anatomical structures are defined as contiguous collections of voxels, each voxel assigned an $x-y-z$ coordinate (Table I). Though this scheme was intended for the Talairach Atlas, its design was purposely general so that it can be used as a guideline for labeling other atlases. The general scheme is to subdivide a structure at level $\mathrm{N}$ into its component substructures in level N-1. For example, the brain is subdivided into its component volumetric structures (cerebrum, cerebellum, and brainstem) in level 1. Likewise, the cerebrum (in level 1) is subdivided into its component structures (lobes and sublobar regions) in level 2 . This scheme continues down to level 5 for the Talairach Atlas. Failure to reach level 5 indicates the bounding level of the labeling scheme for a given structural component. For example, there are no level 5 structural components for white matter (WM) or cerebral spinal fluid (CSF), since no subcomponents are resolvable in the Talairach Atlas. The naming hierarchy in Table I does not illustrate the full detail of names used for lobes, gyri, nuclei, and subnuclear structures (see Talairach Daemon at http://ric.uthscsa.edu/projects for more details).

\section{Talairach Atlas segmentation}

A volumetric image was synthesized from the Talairach Atlas with 1- $\mathrm{mm}^{3}$ voxels such that each voxel can be addressed using Talairach coordinates. Axial section images from the atlas were digitized using an Hewlett Packard ScanJet IIcx color scanner at a resolution of approximately $0.1 \mathrm{~mm}$. Each brain structure (or region) was automatically extracted using thresholding techniques and manually detailed to correct for errors. Labeled regions were reduced to a spatial resolution of $1 \mathrm{~mm}$. Nearest-neighbor interpolation was used to provide data between the atlas sections. Region labeling and extraction started at level 1 of the labeling scheme by forming the cerebrum, cerebellum, and brainstem into 3-D volumes of 
interests (VOIs) using Alice ${ }^{\mathrm{TM}}$ and Dipstation ${ }^{\mathrm{TM}}$ image processing software from the Hayden Image Processing Group (Boulder, CO). Common boundaries of VOIs were used in the creation of VOIs in each successive level. This greatly simplified the creation of new VOIs and was consistent with the volume hierarchy labeling scheme.

Preliminary rules for boundary definitions were developed where boundaries were not explicitly given in the atlas (e.g., Brodman areas) [Freitas et al., 1996]. These rules have been greatly refined and are given for structures within each hierarchical level of the cerebrum as follows:

Level 1 (Hemisphere)

Exterior boundaries defined using a convex hull of cortex

Medial boundaries manually outlined from atlas sections

Level 2 (Lobe)

Lobes

Exterior boundary identical to hemispheres

Interior boundary formed by lines connecting deepest extremes

Sublobar (a single sublobar region was defined to fill remainder of volume within the hemisphere)

Level 3 (Gyrus)

Gyri

Exterior boundary was identical to associated lobe

Interior boundary formed by lines connecting deepest points of limiting sulci

Intragyral boundaries formed by lines bisecting limiting sulci

Minor invaginations (without atlas labels) were ignored

Subgyral (a single subgyral region was defined to fill remainder of volume within lobes)

Nuclei (manually labeled following atlas)

Extranuclear (a single extranuclear region was defined to fill remainder of sublobar volume)

Level 4 (Tissue Type)

Gray matter (GM) and white matter (WM) are readily bounded within gyri

WM and cerebral spinal fluid (CSF) are readily bounded within subgyral regions; the CSF is further labeled by ventricle

GM and WM are readily bounded within nuclei WM and CSF are readily bounded within extranuclear regions

Level 5 (Cytoarchitecture)

Brodmann areas (Interior and exterior boundaries are constrained to cortical GM; Brodmann intergyral boundaries are taken at the pit of associated sulcus; Brodmann intragyral boundaries selected to maintain continuity with other sections where landmarks are available)

Subnuclear structures are readily bounded 


\section{Talairach Daemon}

A unique 3-D coordinate-based database structure was developed for serving brain labels. The core of the database is a 3-D image array spanning the full extent of Talairach space at a resolution of $1 \mathrm{~mm}$ with $\mathrm{x}-\mathrm{y}-\mathrm{z}$ dimensions of $170 \times 200 \times 210 \mathrm{~mm}$. Each $1-\mathrm{mm}^{3}$ voxel within this 3-D array contains a pointer to a voxel record that provides information about labels for that voxel. Each voxel record contains pointers to labels. This scheme provides rapid indexed access to any label within this space. The database is accessed through a server that accepts requests, performs the requested query, and sends results to the requesting application. Both the database and server are memory-resident and run on a Sparcstation 20 Sun Microsystems (Mtn. View, CA). The database and server are called the Talairach Daemon (TD) to indicate the brain standard used and the memory-resident nature of the software. By design, database transactions can be performed at extremely high speeds. High throughput is achieved by keeping the query and response data stream small and using tcp/ip sockets for communications.

\section{RESULTS}

The volume-occupancy labeling scheme was used to guide the bounding of the Talairach Atlas for all level 1 structures as well as cerebrum structures for levels 2-5. One hundred sixty structures have now been incorporated into the Talairach labels database served by the Talariach Daemon. Level 4 is not fully labeled, but this work is underway. Segmentation and label designation for levels 2-5 for the cerebellum and brainstem are planned in the future.

The first-generation Talairach Daemon (TD) is now fully operational. A Java applet is now available (http://ric.uthscsa.edu/projects) and provides easy access to labels and overlays of structures in the TD databases. An overview of the database and server is available at this site. The Talairach Daemon also provides access to structure probability maps (SP maps). In fact, it was specifically designed to provide access to the more complex probability maps. These data are a product of a P20 project seeking to develop a probabilistic reference system for the human brain [Mazziotta et al., 1995]. Currently nine structures are in the SP maps database. The SP maps provide a probability of occurrence for each structure, given an $x-y-z$ coordinate. Graphic overlays are provided for each structure in the Talariach labels and SP maps databases. The overlays from the SP maps represent the regions where the SP map for a structure is $50 \%$ or larger.

Figure 1 is taken from the atlas view window of the TD Java applet. It illustrates overlaying of several lobes onto an electronically modified version of 1988 Talairach Atlas axial section images. The TD applet also provides interactive access to Talairach labels, formatted using the hierarchical volume-occupancy scheme, as well as SP map labels and probabilities. As the pointer is moved around in the atlas window, $x-y-z$ coordinates are displayed on the main window. Labels can be fetched by clicking the mouse button while pointing at a site of interest in the atlas window. The requested labels are printed to the main window with good time response for most users (1-4 sec for most sites around the world).

\section{DISCUSSION}

This preliminary report expands a recently developed volume occupancy naming strategy and describes rules developed for segmentation and labeling. The new volumetric segmentation rules and labeling scheme provide a natural method to hierarchically organize and label a 3-D brain atlas. The accuracy of the Talairach labels is dependent on the accuracy of the spatial normalization used to conform brain images to the 1988 atlas brain. For spatial normalization methods used today, it is anticipated that label conformance will 
be good for structures in levels 1-3. The degree of label conformance will diminish in level 4, but should be better for higher degree-of-freedom spatial normalization methods [Collins et al., 1994; Friston et al., 1995]. Finally, in level 5, where current spatial normalization schemes fail, the labels should be used with caution. A critical evaluation of the accuracy and precision of this label serving scheme is underway [Freitas et al., 1996] in highresolution MR images spatially normalized using a modality-independent 9-parameter spatial normalization method [Lancaster et al., 1995].

Plans are under development to incorporate the volume occupancy naming scheme into the BrainMap database using the Talairach labels database. Our experience with the BrainMap database indicates that the anatomical labels from the Talairach Daemon should be more standardized (and therefore more useful in queries) than conventional anatomical descriptions found within the human brain mapping literature. This is a good example of the use of the forward-transform method for accessing anatomical labels. An example of using the inverse-transformation for labeling is seen with the Talairach Daemon applet, where the various anatomical region labels are overlaid onto the Talairach Atlas section images to show the full extent of region labels.

The Talairach Daemon is currently accessed by numerous sites around the world using the Java applet. Additionally, access to the Talairach labels and SP maps databases is provided in the most recent release of the BrainMap Search and View software (version 4.0 for Sun Solaris is available for download at our website). We are currently evaluating software for direct access to the TD database using TD routines incorporated into in-house software and as stand-alone command-line programs. Final versions of these TD routines will be available at our website in the future.

\section{Acknowledgments}

Contract grant sponsors: NIMH and NIDA; Contract grant number: Human Brain Project P20 MH/DA52176.

\section{REFERENCES}

Collins DL, Neelin P, Peters YM, Evans AC. Automatic 3D intersubject registration of MR volumetric data in standardized space. J Comput Assist Tomogr 1994;18:192-205. [PubMed: 8126267]

Collins DL, Holmes CJ, Peters TM, Evans AC. Automatic 3-D model-based neuroanatomical segmentation. Hum Brain Mapping 1995;3:190-208.

Fox PT. Spatial normalization origins: Objectives, applications, and alternatives. Hum Brain Mapping 1995;3:161-164.

Fox, PT.; Mikiten, S.; Davis, G.; Lancaster, JL. BrainMap: A database of human functional brain mapping. In: Thatcher, RW.; Zeffiro, T.; Huerta, M., editors. Advances in Functional Neuroimaging: Technical Foundations. Academic; Orlando: 1994. p. 95-106.

Freitas CS, Summerlin JL, Lancaster JL, Fox PT. Talairach Daemon: Automated mapping of surface anatomy onto standard coordinates. Society for Neuroscience 1996;22:675.

Friston KJ, Ashburner J, Frith CD, Polint JB, Heather JD, Frakowiak RSJ. Spatial registration and normalization of images. Hum Brain Mapping 1995;3:165-189.

Lancaster JL, Glass TG, Lankipalli BR, Downs H, Mayberg H, Fox PT. A modality-independent approach to spatial normalization of tomographic images of the human brain. Hum Brain Mapping 1995;3:209-223.

Mazziotta JC, Toga AW, Evans A, Fox P, Lancaster J. A probabilistic atlas of the human brain: Theory and rationale for its development. Neuroimage 1995;2:89-101. [PubMed: 9343592]

Roland PE, Graufelds CJ, Wahlin J, Ingleman L, Andersson M, Ledberg A, Pederson J, Akerman S, Dabringhaus A, Zilles K. Human brain atlas: For high-resolution functional and anatomical mapping. Hum Brain Mapping 1994;1:127-184. 
Talairach, J.; Tournoux, P. Co-Planar Stereotaxic Atlas of the Human Brain. Thieme Medical Publishers, Inc.; New York: 1988. 


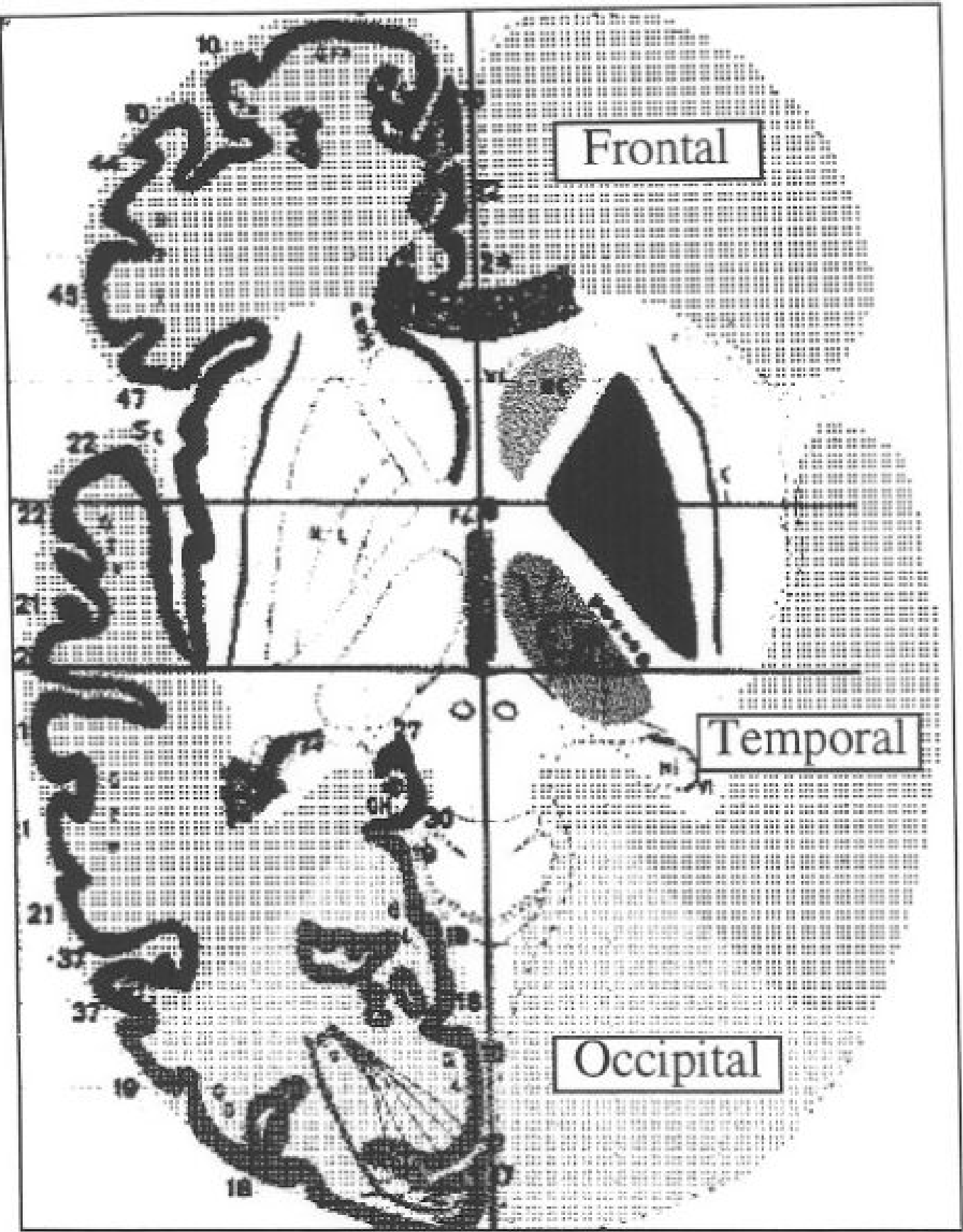

Figure 1.

Overlays of Talairach labels for frontal, temporal, and occipital lobes for the transaxial section image from the Talairach Atlas at $\mathrm{z}=+1$ (background adapted from Talairach and Tournoux [1988]). 
